\title{
RESPIRARE A DUE POLMONI L'ICONA E LA SUA POSSIBILITÀ DI INSERIMENTO NEI LUOGHI DI CULTO CATTOLICI
}

Gianluca Busi

Facoltà Teologica dell'Emilia Romagna

\begin{abstract}
The author, a Catholic priest, iconographer as well a member of a commission for the sacred fine art in Italy, traces starting from the Pope's Benedict XVI book The spirit of the liturgy some lines, resumed in five thesis, about the possibity in the catholic Church to reinstate a sacred fine art looking back to the eastern lost treasures.

Considering the catholic churches built after the'60th he divides them in 4 major categories: churches not accept inside any figurative image, the ones introduces only artistic stained glass patterns, the ones hosts figuratives images but without respect for the traditional figurative canon and the ones accept figurative but canonical images/icons.

At the end he try to reach this point of view: even by the catholic tradition after the middle age and even more after the mannerism is tipically opened to any kind of art (even the abstract) it will be interesting to promote today new works through canonical/icons images. If this will happen artistic projects will to be respectful with the idea to breath with two lungs, for a brand new way to reshape the sacred fine art watching at the eastern and the western tradition. To paint icons and iconografic projects for the churches can be a plausible way to set the new catholic art in the 3rd millenium.
\end{abstract}

Key words: iconographer, icons, liturgy, church, tradition

L'Introduzione allo Spirito della liturgia, testo tradotto in italiano nel 2002, riassume le posizioni maturate nel corso della lunga attività di teologo e pastore del cardinale divenuto papa con il nome di Benedetto XVI e si iscrive nella riflessione già portata avanti dal suo predecessore san Giovanni Paolo II, che attraverso la Lettera apostolica del 2 maggio 1995, invitò la chiesa Cattolica a riconsiderare il patrimonio della cosiddetta "Chiesa indivisa del primo millennio", quale respiro a due polmoni, l'orientale e l'occi-

e-mail: sleodgianluca@alice.it 
dentale, per ristabilire un equilibrio che la Chiesa cattolica avrebbe perduto progressivamente .

L'Introduzione allo Spirito della liturgia è un esempio per l'ampiezza delle coordinate di riferimento, lo chiarezza dello sviluppo e la forza delle conclusioni teologiche tratte.

L'obiettivo che mi propongo è capire quale sia la posizione di un eminente teologo sull'iconografia canonica nella Chiesa oggi. Immagino inoltre di poter capire, alla luce della mia esperienza personale di pittore di icone, quali siano concretamente gli esempi a cui egli si riferisce nel libro e cerco di codificarle attraverso categorie visuali.

Arrivo infine ad alcune conclusioni in cui accenno a quello che, secondo il mio modesto punto di vista, potrebbe essere il nodo centrale della questione: cioè quale collocazione e plausibilità possano avere le opere d'arte figurativa e in particolare le icone nei luoghi di culto di cattolici di tradizione latina.

L'Introduzione allo spirito della liturgia è un testo piacevolmente leggibile, snello e molto chiaro e si distingue per l'essenziale brevità: appena centocinquanta pagine. Ma ancora più breve è la parte che riguarda il tema preso in esame (il terzo capitolo): in tutto una trentina di pagine. Il capitolo prende il titolo di Arte e Liturgia. ${ }^{l}$

Il testo di riferimento, benché scritto un decennio prima, è stato inserito pressoché immutato nel volume undicesimo dell' $O$ pera Omnia di Joseph Ratzinger, con il nuovo titolo di Teologia della Liturgia. Il corpo del testo lascia intuire un pensiero maturo sull'argomento, che non necessitava di revisioni o post-fazioni di sorta.

Prima di entrare nell'analisi del pensiero del cardinale occorre fare un precisazione necessaria: la collocazione delle immagini è una questione dibattuta nella Chiesa cattolica dal medioevo in poi, in particolare dal periodo seguente il Concilio Lateranense IV del 1215 e l'istituzione della Solennità del Corpus Domini del 1265.

\footnotetext{
${ }^{1}$ Joseph Ratzinger, Opera Omnia, 11: Teologia della Liturgia. Introduzione allo spirito della liturgia, 11 voll. (Città del Vaticano: Ed. Vaticana, 2010), 114-132. Mi concentro, per via del contesto prossimo al tema preso in considerazione, sul capitolo Arte e Liturgia.
} 
Non è cioè una sorta di invenzione concepita negli ultimi trent'anni in seguito alla rinascita dell'iconografia canonica trasmessa alla Chiesa cattolica soprattutto attraverso l'esperienza e l'impulso recente dalla Chiesa russa.

Capire quale posto e statuto abbiano le immagini è un problema che affonda le radici nella tradizione della Chiesa di rito e ambito latino. Nelle diverse epoche infatti i vari documenti ufficiali hanno ripreso questa tematica e nelle tappe dell'evoluzione del dogma hanno ribadito la medesima posizione nell'arco dei secoli. ${ }^{2}$

Mi interessa sottolineare questo aspetto: c'è una tradizione ed una evoluzione del dogma, tuttavia si è sempre optato in ambito cattolico per "Non prediligere uno stile rispetto ad un altro, per le immagini dedicate al culto", il testo è fra virgolette perché è una parafrasi di un testo del Vaticano II. Questa è la posizione tipicamente cattolica quando si tratta di formulare una teologia dell'immagine/icona.

Il teologo Ratzinger sorprende per l'eccezionale competenza della materia, se si considera che l'ambito della sua ricerca si sviluppa altrove. Questo si deve probabilmente al fatto che egli è da sempre un cultore delle icone a titolo personale, e va ricordato ad esempio che, su invito della professoressa Maria Giovanna Muzj dell'Università Gregoriana, egli accettò nel 2001 l'invito a tenere la prolusione per il primo incontro degli iconografi italiani a Roma quale segno di attenzione e predilezione per questa materia.

Come si compone il breve capitolo dello Spirito della liturgia che prendo in esame Essenzialmente in tre grandi parti. Un'ampia introduzione, in cui l'autore individua le tappe salienti della teologia dell'icona in occidente.

Una prima parte, in cui si pone la domanda se la teologia dell'icona mediata dal secondo concilio di Nicea del 787 sia valida anche per l'oggi in ambito latino (una domanda che si centra sul nodo fondamentale della questione).

\footnotetext{
${ }^{2}$ Cf. l'esaustiva nota 51 di G. Busi, Il Segno di Giona, 61, in cui prendo in considerazione le posizioni del Magistero espresse nei diversi documenti nell'arco dei secoli, a partire dal Concilio Costantinopolitano IV (869-871), fino alla costituzione Orientalium Ecclesiarum (1964).
} 
Una seconda parte, ancora più interessante, in cui egli si chiede come proseguire positivamente questo dibattito nel contemporaneo, messa a fuoco attraverso cinque tesi di fondo che egli puntualizza per definire quella che egli chiama Arte ordinata alla Liturgia. Proprio l'analisi delle tesi sarà il punto centrale su cui mi soffermerò per le mie valutazioni in merito.

Parlare di arte ordinata alla liturgia richiede una riflessione aggiuntiva per capire come definire un ambito di ricerca: il cardinale cioè non è immediatamente interessato ad una categoria ampia quale la cosiddetta arte religiosa (che potrebbe definirsi come aiuto per elevare la mente verso una spiritualità generica) né della cosiddetta arte sacra (definibile come ambito del sacro). Con questi termini si intendono infatti ambiti molto più ampi se riferiti al contesto che egli considera in questo testo, cioè quello ben più ristretto dell'arte detta appunto per la Liturgia. Un'opportuna definizione direbbe che egli restringe la sua analisi ad un'arte ecclesiale che si presenta come contestuale ed opportunamente sintonizzata alla celebrazione dei divini misteri.

Ora resta chiaro che l'iconografia canonica che scaturisce dal riferimento con Nicea II sia orientata alla liturgia per definizione, mentre ci si dovrebbe chiedere se arte religiosa e sacra siano plausibili con la liturgia e la sua celebrazione. Quando si tratta di ordinare l'arte allo svolgimento e all'ambito della liturgia che cosa va ricercato? Egli espone il suo pensiero in merito attraverso le cinque tesi che enucleo sinteticamente:

1. Il figurativo per l'arte cristiana è una necessità dipendente dall'incarnazione.

2. I contenuti derivano dalla Historia Salutis.

3. Il mistero del Cristo morto e risorto è al centro dell'arte sacra ed esso non va mai diviso. ${ }^{3}$

\footnotetext{
${ }^{3}$ L'equilibrio fra la divino-umanità, acquisizione fondamentale che si richiama alle affermazioni del Concilio di Calcedonia, è fondamentale per una cristologia esatta. Conosciamo infatti quei tentativi delle cosiddette cristologie del Novecento, in cui molto spesso si evidenzia un evidente squilibrio fra divinità ed umanità. Evidenziare un Cristo totalmente sofferente come nelle cosiddette cristologie della morte di Dio, relativizza l'aspetto divino e rende troppo orientato al dramma
} 
4. Le immagini devono riferirsi alla teologia del secondo Concilio di Nicea. ${ }^{4}$

5. Questa tesi è quella che lascia più perplessi. Il cardinale afferma infatti che benché debba far teologicamente riferimento al secondo concilio di Nicea, la Chiesa di occidente non deve rinnegare il cammino che ha intrapreso dall'affermazione del Gotico. Viene indicata qui la frattura che esiste all'interno della Chiesa dopo il primo millennio, o meglio dopo il 1204, data della quarta crociata che definisce lo spartiacque che divide le due tradizioni. Considerando che l'inizio del gotico si data normalmente al 1240 con l'erezione della rinnovata abbazia di Saint Denis a Parigi, si legge una quasi coincidenza fra i periodi. Riprende il cardinale:

Questo cammino tuttavia dovrebbe ricentrarsi sulle coordinate stabilite nel secondo Concilio di Nicea, senza però assumere tutte le norme del canone ed aggiunge, così come è avvenuto ad esempio in Russia nel Concilio dei 100 Capitoli del 1551. ${ }^{5}$

Cosa successe precisamente nel concilio moscovita dei Cento Capitoli? Si potrebbe dire sinteticamente che in quella occasione la Chiesa russa si è appropriata delle regole per un'arte dedicata alla liturgia. Ha così istituito l'iconografia come ministero all'interno della Chiesa, a sua volta regolato da norme canoniche. Definisce anche come deve essere un dipinto a favore della liturgia, poiché la Chiesa ne regola il canone, ne stabilisce i criteri e giudica sulla eventuale compatibilità.

Si potrebbe dire che nello Stoglav' la Chiesa russa si è attribuita il potere di assegnare un canone all'iconografia e all'icono-

il Mistero, mentre si banalizza l'aspetto umano se lo si descrive con espressioni troppo orientate alla risurrezione già realizzata (come potrebbe essere un'icona del Cristo buonista figlio dei fiori).

${ }^{4}$ Questa affermazione sorprende molto positivamente per chi si muove partendo dalle riflessioni della chiesa orientale, che non ha mai abbandonato questo punto di vista. Il Niceno II infatti non è altro che una traslazione degli stessi temi espressi nei concili cristologici dei primi secoli riformulati riguardo al tema dell'iconografia.

${ }^{5}$ Joseph Ratzinger, Introduzione allo spirito della Liturgia (Torino: San Paolo Edizioni 2014), 131. 
grafo, cosa che invece non è mai avvenuta nella tradizione occidentale, malgrado alcuni tentativi in questa direzione ad opera dei cardinali Bellarmino e Paleotti nel contesto della Controriforma (emblematica in questo contesto è la richiesta fatta al discepolo di Michelangelo, Daniele da Volterra il braghettone, di coprire con delicati panneggi le nudità di alcuni personaggi nella Cappella Sistina. Questi ed altri interventi furono commissionati dall'autorità ecclesiale per arginare fenomeni devianti dallo spirito della liturgia avvenuti nel manierismo).

Il cardinale afferma che il cammino che parte dal gotico non va dimenticato, tuttavia va purificato ricentrandosi sulle coordinate del secondo Concilio di Nicea. Cosa intenda in concreto con purificazione non è purtroppo così evidente nel testo e lascia pensare ad un accenno che richiederebbe un'ulteriore riflessione su cui il testo non va oltre. Di fatto egli si limita a riaffermare la via tipicamente cattolica del non preferire uno stile espressivo rispetto ad un altro.

Questo indica a mio parere la differenza che intercorre fra la modalità orientale, che regola l'arte liturgica attraverso il canone al contrario della modalità occidentale che non ha mai espresso uno stile canonico propositivo-impositivo, quanto piuttosto si è limitata ad indicare percorsi correttivi, per purificare appunto le derive che si sono via via presentate durante i secoli.

Considerati questi elementi, passo ora a considerare il che cosa immaginava il teologo Ratzinger quando scriveva le sue cinque tesi. Egli, come cardinale arcivescovo della diocesi di Monaco e poi successivamente come vescovo di Roma, ha visitato innumerevoli chiese ed ha visualizzato innumerevoli progetti iconografici in tutto il mondo: quando scriveva lo Spirito della liturgia poteva attingere dai suoi ricordi cicli di immagini specifici, visti di persona.

Prendendo spunto da questa congettura mi propongo una rilettura squisitamente personale del testo alla luce di alcune opere contemporanee nei luoghi di culto cattolici.

La mia rilettura confronta le cinque tesi appena esposte con alcune opere monumentali realizzate negli ultimi anni per chiese cattoliche sparse in tutto il mondo. A partire dalle conclusioni del testo, e in particolare dalla quinta tesi (che mi sembra la più interes- 
sante fra tutte), vorrei osservare l'esistente e ricavare le domande giuste per comprendere i nodi salienti del dibattito teologico circa le immagini per la Liturgia nella tradizione occidentale.

Divido le opere iconografiche in quattro amplissime categorie. Esse sono come i punti cardinali, chiaramente identificabili una volta che si conoscano abbastanza le produzioni contemporanee e le si voglia codificare. Le riassumo in un breve elenco:

- $\quad$ opere non figurative (pura astrazione);

- $\quad$ opere non figurative che si avvalgono di registri figurativi (tipico esempio: le vetrate istoriate);

- $\quad$ opere figurative che si ispirano all'arte contemporanea ma che non sono sottoposte ad un canone;

- $\quad$ opere figurative idealmente sottoposte al canone.

L'elenco potrebbe essere ulteriormente sintetizzato nei due grandi ambiti del non figurativo (1 e 2) e del figurativo (3 e 4).

Leggendo lo Spirito della liturgia si avverte fra le righe una avversione implicita per le prime due categorie che identificano le opere attraverso registri non figurativi. Un aspetto che stupisce molto se si considera che nelle chiese contemporanee queste due categorie sono quelle più utilizzate, e che innesca una domanda molto seria circa la plausibilità di molte opere realizzate nel contemporaneo per la liturgia. All'interno del dibattito odierno, mi sembra che proprio il criterio della plausibilità plasmi quella prima domanda che è opportuno porsi. Di seguito traduco, attraverso descrizioni supportate da immagini, le categorie per rendere fruibili ai non addetti ai lavori le sfumature di questo argomento.

\section{Non figurativo}

Parlare di opere d'arte non figurative per la liturgia può sembrare un paradosso per chi conosce la tradizione. In realtà si tratta di prendere in considerazione soprattutto quei luoghi di culto contemporanei che si potrebbero chiamare olistici. Queste opere recenti, infatti, presuppongono una concezione particolare per la quale la forma stessa dell'edificio e la qualità dei materiali vorrebbero essere esaustivi per definire un luogo di culto, quale opera d'arte conchiusa. Per favorire una comprensione più immediata, indico 
alcuni luoghi di culto costruiti in ambito internazionale, nell'arco dell'ultimo decennio.

Punto di partenza obbligato è la chiesa protestante di Ibara, in Giappone, ad opera di Tadao Ando. Sul fondo dell'aula, essenzia-

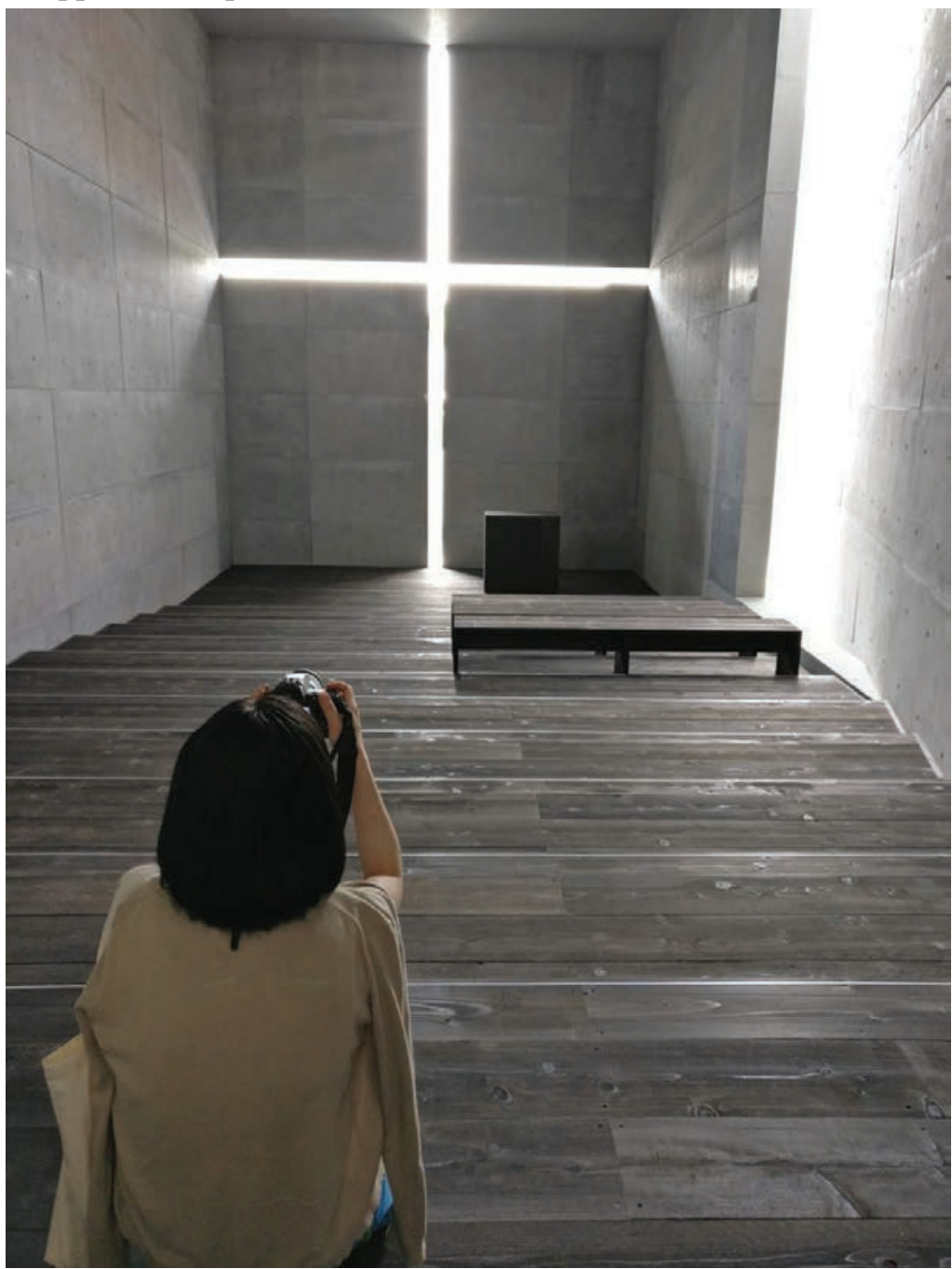

Fig. 1. Chiesa protestante di Ibara, Giappone, ad opera di Tadao Ando 
le e di forma cubica, si staglia una croce disegnata dalla luce che scaturisce dalle fessure nei muri. Prendo in considerazione come emblema di questa particolare categoria questa chiesa perché vorrei indicare come questo registro dell'aniconicità, molto diffuso in ambito cattolico a partire dalle realizzazioni-pilota di Le Corbusier e di Michelucci trovino un riferimento genetico e consueto nelle chiese della riforma.

Questi edifici hanno infatti alle spalle cinque lunghi secoli di tradizione aniconica ed anche quando introducono un segno apparentemente figurativo (il crocifisso) lo fanno togliendone il corpo ed introducendo un'immagine non figurativa ma essenzialmente simbolica.

La chiesa di recentissima costruzione progettata dall'architetto Massimiliano Fuksas a Foligno, vincitrice di un concorso internazionale è un edificio dal doppio volume; quello interno si solleva di circa due metri dal pavimento. Oltre ai fuochi liturgici non vi è alcuna iconografia, nell'abside non vi è alcuna figurazione. Ma c'è un motivo, ancora una volta olistico: qui l'architettura vorrebbe essere l'espressione artistica unica, in grado cioè di assorbire ed esaurire ogni altra espressione artistica del luogo di culto, quali una $o$ più opere di arte figurativa consuete.

Ci si chiede se questa chiesa costruita di recente a Vienna sia un luogo di culto protestante, considerata l'affinità con la citata opera di Tadao Ando. Ma, al contrario, di tratta di una chiesa cattolica in cui l'abside rappresenta semplicemente, attraverso le fenditure praticate nel corpo dell'edificio, una croce che si illumina quando viene attraversata dalla luce proveniente dall'esterno. Possiamo incontrare un'infinità di chiese analoghe fra le nuove costruzioni, tanto è diffuso nella chiesa cattolica questo nuovo modo di intendere gli edifici di culto.

Nella Chiesa del Giubileo di Meier nel quartiere di Tor Tre Teste a Roma, la struttura esterna è composta da tre grandi vele, che vengono chiamate anche mani, interpreta ancora una volta il desiderio olistico di un'architettura che nega ogni figurazione al suo interno. Interessante notare come nell'abside della navata centrale, nata come elemento non figurativo, sia stato giustapposto in epoca posteriore un crocifisso che, se da una parte non si integra per nulla 
nello spazio, dall'altra dà voce al disagio di una comunità che vive e celebra di fronte a impianti non figurativi.

La recentissima cattedrale di Oakland, negli Stati Uniti una delle costruzioni più grandiose del decennio per dimensioni e sforzo progettuale, propone una curiosa mescolanza di figurazione e non figurazione all'interno di un colossale impianto architettonico, che deve la sua forza a un muro ricurvo composto di lamelle lignee attraversate dalla luce. Lascia davvero perplessi considerare l'inserimento nell'abside di un gigantesco corpo vitreo, aniconico al buio ma che permette invece in presenza di luce una lettura in filigrana da cui appare nella sua maestosità una immagine di Cristo in trono

Questa breve rassegna, che ritengo sufficiente per comprendere la problematica attuale, trova il suo cuneo ermeneutico nella Cattedrale di Oakland, esempio emblematico per il tentativo iperbolico di passaggio dalla non figurazione alla figurazione attraverso la assenza-presenza della luce. Inoltre cerca di dire e non dire proponendo una sorta di via mediana in cui ogni opzione è possibile.

Tentativo di adesione all'aniconismo contemporaneo che riconosce al contempo la necessità di misurarsi con la figurazione perché interna alla tradizione dei luoghi di culto (in cui sempre la divino-umanità è stata rappresentata attraverso categorie figurative), questa cattedrale enuclea in modo esemplare la difficoltà della categoria appena descritta.

\section{Non figurativo (vetrate)}

Inserisco in questa categoria un'ulteriore distinzione rispetto a quella presentata al punto uno. La specificità si deve al fatto che la vetrata istoriata risale al periodo gotico e ne assume i significati concettuali peculiari. La vetrata gotica, il cui emblema è l'impianto della cattedrale di Chartres del XII secolo, è chiaramente figurativa. Se uno entrasse in una di queste cattedrali avvalendosi di una guida illustrata, la fotografia dal libro gli permetterebbe di dedurre immediatamente la parte grafica della vetrata e capire che l'impianto è assolutamente figurativo con un livello di dettaglio artistico e didattico a dir poco sorprendente.

Tuttavia, quando si entra in queste cattedrali, la prima cosa che si coglie sono delle macchie di luce. Può darsi che questo aspetto 
sfugga ai più, ma non si può negare di trovarsi di fronte ad un aspetto paradossale: possiamo dire infatti che le vetrate sono figurative e nello stesso tempo non lo sono immediatamente, poiché si presentano appunto, quando invase ed attraversate dalla luce, come splendidi pattern cromatici.

Prima ancora di presentare l'aspetto figurativo, come farebbe ad esempio un affresco, la vetrata istoriata concepita per le cattedrali gotiche impone una comunicazione di ordine concettuale e, di conseguenza, non figurativa. ${ }^{6}$

L'idea che presiede la concezione delle vetrate istoriate può riassumersi così: la luce che proviene dall'esterno, cioè dal mondo, non potrebbe entrare nello spazio sacro senza essere educata, convertita, dalla predicazione evangelica. Deve cioè sottostare allo stesso principio cui sottostava il fedele nel medioevo quando, nell'atto di entrare nella cattedrale, era chiamato a passare dal mondo che culminava nel sagrato antistante l'edificio al tempio dove Dio prende dimora. In questi edifici il portale offre al fedele, attraverso cicli teofanici e narrativi, una catechesi obbligatoria per attraversare la porta d'ingresso con una cognizione opportuna.

È indispensabile per un turista di oggi rivisitare queste acquisizioni, allora così naturalmente conosciute e percepite e di cui nell'epoca attuale sono andati perduti quasi completamente i codici ermeneutici. Se riacquisiti, costituiscono per l'osservatore odierno quel thesaurus di informazioni che permette di comprendere il contesto nativo delle opere d'arte. Le vetrate nascono certamente come figurazioni ma parlano prima ancora il linguaggio del non-figurativo, poiché la prima e fondamentale percezione che offrono è quella di un pattern cromatico che tocca il visitatore risvegliandone la sensibilità estetica. Presento brevemente quattro luoghi di culto che mostrano la diffusione e il reimpiego della vetrata nel contemporaneo.

Una chiesa a Monaco di Baviera presenta un impianto vetrato che raggiunge le stesse dimensioni dell'abside, tanto da dominarlo

\footnotetext{
${ }^{6}$ Riferimenti più precisi ed esaurienti sulle vetrate istoriate, sulla loro collocazione e la peculiare visione teologico-catechetica sono reperibili nell'ottimo testo di Xavier Barral i Altet, Vetrate medievali in Europa (Milano: Jacabook, 2003).
} 


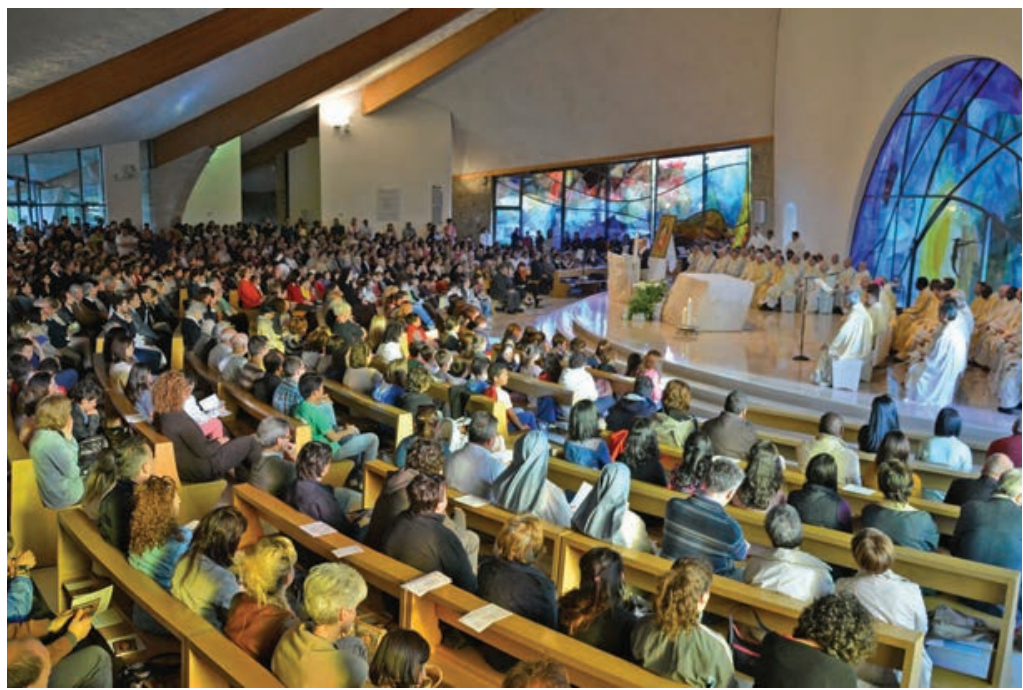

Fig. 2. Chiesa della Theotokos a Loppiano, Firenze

completamente. Spicca in questo contesto un piccola croce bianca collocata alla spalle dell'altare.

La grande chiesa della Theotokos a Loppiano, sede del movimento focolarino fondato da Chiara Lubich, presenta diverse istallazioni vetrate a tema mariano. Nella chiesa del Kaiser Memorial di Berlino un'amplissima superficie vetrata di colore azzurro fa da sfondo cromatico ad un crocifisso appeso.

Per concludere la carrellata accenno ad chiesa brasiliana che presenta uno degli aspetti più fantascientifici. Le vetrate non sono collocate, come è la norma, nel fronte dell'abside ma seguono il profilo della copertura. Composte da file di piccoli riquadri, alternano vetri colorati secondo la sequenza del caleidoscopio ed in presenza della luce illuminano di riflesso l'abside di mattoni a vista, creando una potente suggestione cromatica.

$\mathrm{Si}$ intuisce facilmente che le vetrate artistiche menzionate in questi esempi richiamandosi all'impianto delle cattedrali del periodo gotico, hanno lo scopo di suscitare una forte emozione di tipo metafisico-religioso. 
Se dovessimo limitarci alle tesi esposte nell'Introduzione allo spirito della liturgia, si potrebbe dire che le chiese al punto 1 siano non compatibili mentre, per quello che riguarda la specificazione della vetrata nel punto 2 , forse si potrebbe formulare un giudizio più possibilista $\mathrm{e}$ valutarle con maggiore clemenza.

\section{Figurativo non canonico}

Dopo aver descritto per sommi capi la situazione odierna relativa al primo grande ambito di ricerca, che si riassume nelle prime due categorie dell'astrazione e delle vetrate intese come pattern non-figurativi, prendo in considerazione il secondo ambito dedicato al figurativo e che costituisce la parte più interessante e contestuale della mia indagine.

L'ambito del figurativo si compone idealmente di due grandi categorie il cui discriminante è il cosiddetto canone. Nessun termine è più equivoco in materia di storia dell'arte, limitatamente a questo contesto ne considero la definizione classica, secondo cui si dice canonica l'opera che appare perfetta e armoniosamente proporzionata perché plasmata da norme orientate all'equilibrio compositivo.

Di conseguenza, con la categoria del figurativo non canonico indico quel particolare filone artistico contemporaneo che si esprime attraverso la figurazione nello spirito di una certa evoluzione ed interpretazione del canone. Come di consueto presento opere ed artisti noti per illustrare questo punto.

Marko Ivan Rupnik, gesuita di origine slovena, co-fondatore del centro Aletti di Roma, è uno degli artisti più noti e rappresenta con la sua opera una delle espressioni di punta della nuova arte figurativa emersa nel post-concilio. Gode di immensa stima negli ambienti cattolici, lo stesso papa Giovanni Paolo II gli affidò il compito di affrescare la propria cappella privata, che egli completò con un impianto musivo che occupa l'intera superficie murale.

L'artista, noto come professore e pubblicista, ha descritto in maniera efficace il percorso personale che lo ha portato a concepire questa nuova forma figurativa. Le forme che egli propone sono frutto di una ricerca molto profonda che parte dalla conoscenza 
delle opere tradizionali e dialoga con le forme artistiche contemporanee per produrre una nuova iconografia. ${ }^{7}$

La cappella Redemptoris Mater, in Vaticano è dipinta negli ambienti attigui alla stanza della Segnatura, ex-biblioteca del papa che fu affidata a Raffaello Sanzio e venne decorata dall'urbinate con affreschi universalmente noti quali la Scuola di Atene e la Disputa del Sacramento. Non deve sfuggire la stima con cui un papa ha affidato un incarico che venne dato a uno dei più grandi artisti di tutti $i$ tempi.

L'iconografia di Rupnik si esprime in maniera emblematica nella composizione dell'impianto musivo absidale della chiesa di monte Argentario. Un impianto figurativo squisitamente classico, in cui ogni buon conoscitore dell'iconografia canonica può scorgere il modulo della Discesa agli inferi con la liberazione di Adamo ed Eva dai sepolcri. Tuttavia il modo di ripresentarlo è critico nei confronti della composizione canonica, di cui destruttura forma e cromatismo, liberandola dal peso di una copia stereotipata della tradizione e ponendola in dialogo con l'arte contemporanea. L'artista ha ripercorso diverse volte questo tema della discesa agli inferi a lui particolarmente ad esempio nella recente realizzazione di un mosaico di $250 \mathrm{mq}$ di superficie per la chiesa del Corpus Domini in un quartiere periferico a Bologna in Italia.

Le forme acquistano dinamicità ed i pattern cromatici, che sono parte costitutiva del mosaico, infondono movimento procurando una emozione religiosa ed estetica in chi guarda. Oggi questo modo di interpretare l'arte sacra per la liturgia è sicuramente in crescita nella chiesa cattolica ed il grado di stima che questo autore rivendica negli ambienti ecclesiali mostra l'accoglienza a questo tipo di percorso, in cui tradizione canonica e libertà interpretativa si fondono.

Altro artista estremamente noto è Kiko Arguello, fondatore del cammino neocatecumenale, che ha affrescato chiese neocatecumenali in tutto il mondo riproponendo nell'area absidale il ciclo della cosiddetta corona misterica che richiama ampiamente lo schema classico delle dodici feste dell'anno liturgico. Al centro troviamo l'iconografia tradizionale dell'episodio evangelico del capitolo ven-

${ }^{7}$ Marko I. Rupnik, Il colore della luce (Roma: Lipa, 2003). 


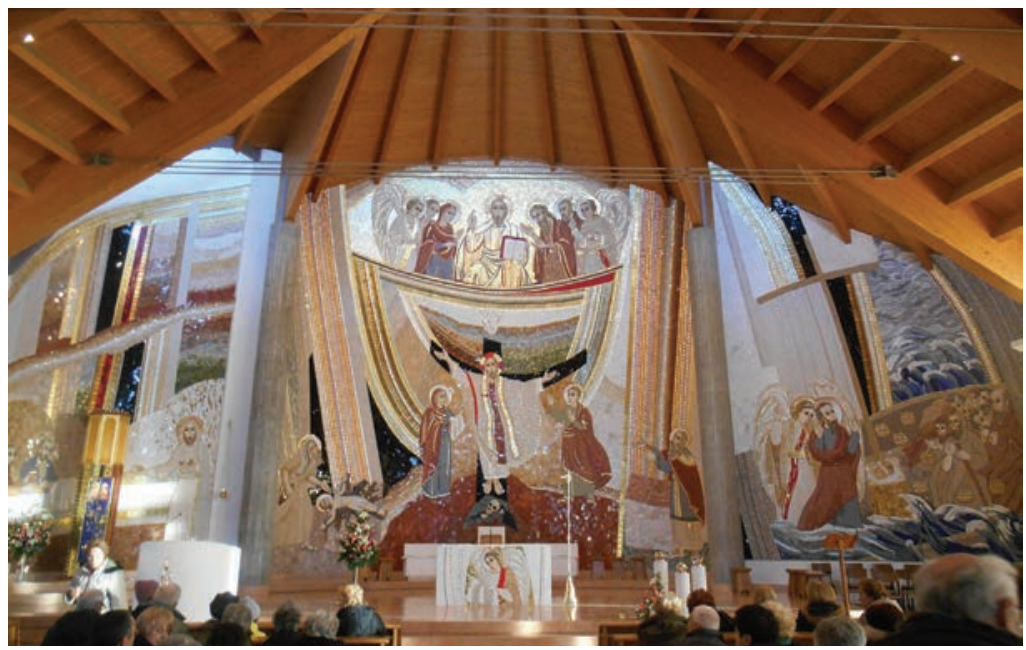

Fig.3. Chiesa del Corpus Domini, Bologna, Marko Rupnik

ticinquesimo del Vangelo di Matteo, in cui Cristo siede sul trono di gloria e opera il giudizio. Come si può notare questo impianto si inserisce nella più squisita iconografia canonica tradizionale, ed è infatti lo stesso presentato nelle iconostasi della tradizione orientale.

A una valutazione formale l'opera di Kiko risulta canonica per i soggetti presentati e per la loro collocazione: fin dalle basiliche protocristiane nell'abside, troviamo la rappresentazione del Cristo giudice ed intorno ad esso altre raffigurazioni che si richiamano ad episodi salienti della storia della salvezza. Se teniamo in filigrana le tesi indicate da Ratzinger, sorprende notare come questi impianti neocatecumenali siano vicini a quanto veniva prospettato come ottimale. ${ }^{8}$

Penso però che a uno sguardo più attento ai dettagli non possano sfuggire alcune imprecisioni. Chi conosce i cromatismi espressi dalla tradizione ad esempio, mi riferisco all'utilizzo di terre e co-

\footnotetext{
${ }^{8}$ Interessante a proposito è il voluminoso e articolato testo in cui si cerca di dare una fondazione teoretica circa l'eccellenza dell'opera di Kiko Arguello in materia di arte sacra: Maurizio Bergamo, Mattia Del Prete, Spazi celebrativi. L'architettura dell'Ecclesia (Bologna: EDB, 2003).
} 
lori naturali e al loro delicato e bilanciato spettro cromatico, non può non notare l'impoverimento derivato dall'uso di colori sintetici dallo spettro aggressivo e non bilanciato, che creano una sorta di eccitazione visiva dissonante - e mi chiedo se questo sia compatibile con il culto, sempre ispirato a forma e misura. Nella misura in cui è permessa un'analogia musicale, sembra di ascoltare un pezzo di Stockhausen paragonato a un corale di Bach.

Anche se qui la forma è più costretta entro il canone di quanto lo sia nei lavori di Rupnik, il cromatismo chiassoso ed aggressivo autorizza a parlare di un figurativo che non è più canonico.

Presento un'opera di Ruberval Monteiro, monaco benedettino brasiliano che sceglie per i suoi affreschi immagini chiaramente ispirate alla tradizione. Ad esempio in questa pittura absidale che incorpora il tabernacolo, viene raffigurato il Cristo giudice dei vivi e dei morti del capitolo venticinque del Vangelo di Matteo, una citazione tradizionale nell'iconografia cristiana per le raffigurazioni absidali. L'artista reinterpreta però figurativamente la tradizione attraverso immagini che sono non canoniche, nel senso che le forme sono rielaborate nella ricerca di un dialogo con il contemporaneo, e con cromatismi e figurazioni che richiamano la pittura arcaica e le atmosfere sudamericane.

Il brasiliano Sergio Ceron propone anch'egli un abside con lo stesso soggetto, il Cristo giudice, contenuto in una mandorla rosso-marrone e contornato dai quattro esseri viventi cioè il classico tetramorfo, con riferimento al capitolo secondo di Ezechiele. Lo schema diventa ancora più canonico perché opportunamente collocato sotto il Cristo troviamo il syntronos per il celebrante, altra squisita citazione dalla tradizione paleocristiana. Tuttavia per la scelta di un cromatismo minimalista e per le forme allungate e non più attinenti la realtà delle proporzioni anatomiche le forme impresse dall'artista tendono ad uscire dagli stilemi classici canonici della figurazione.

Dello stesso artista, un'opera dipinta per la chiesa di Londrina, in Brasile. Ho potuto notare con un certo interesse e una viva inquietudine che questa chiesa, costruita appena dopo il Concilio Vaticano II, presentava un'installazione contemporanea che sembrava richiamare l'immaginario spaziale della saga hollywoodiana 
di Star Wars. soprattutto a causa della luce rossa collocata dietro il tabernacolo e che lo assimila a una supernova. Devo quindi purtroppo segnalare qui lo scarso senso critico con cui si è operato in quegli anni, caratterizzati da un entusiasmo forse troppo indulgente. Questa realizzazione mi sembra un ottimo indicatore per cogliere a quale derive ci si era esposti in ambito artistico.

A questo proposito vorrei far notare che Sergio Ceron ha ricevuto una recente commissione proprio per riformulare un abside che aveva appena trent'anni anni di vita, un sintomo evidentemente positivo che i tempi sono più maturi e che l'arte per la liturgia ha intrapreso una sana forma di autocritica nella ricerca di un equilibrio ed una plausibilità maggiori. L'artista ha ripensato globalmente l'abside e lo ha riformulato in maniera completamente nuova, seguendo quel percorso che gli è proprio e di cui ho già indicato le caratteristiche peculiari. Il fatto che un progetto di ordine figurativo sia stato scelto per sostituirne un altro ispirato al concettuale ed alla forma astratta indica un vibrazione che attraversa la Chiesa cattolica e che esercita una certa pressione perché il figurativo venga rimesso al centro dell'espressione artistica.

\section{Figurativo canonico}

In contrasto con la categoria del figurativo non canonico appena presentata, con quella del figurativo canonico indico un ambito estremamente ampio e variegato che, se descritto nel dettaglio, entrerebbe a fatica in un'unica categoria. Credo tuttavia possibile individuare dei punti comuni tra gli iconografi che presenterò. L'elemento di coesione mi sembra possa essere ravvisato nel tentativo di conformarsi ai modelli tramandati dalla tradizione. Pur nella via di una ricerca squisitamente personale, che per i più presenta $i$ tratti di una originalità marcata, ci sono elementi sufficienti per comprenderli a partire da ciò che li accomuna.

Prendo come punto di partenza l'opera di un iconografo italiano, Paolo Orlando di Trieste. La sua poderosa opera artistica è presente in tutto il mondo. Profondo conoscitore, anche a livello teologico, delle opere e dei testi del passato egli propone un percorso che affonda le sue radici nella patristica e nell'iconografia canonica tradizionale. 
Fra le sue opere più salienti è da ricordare una chiesa parrocchiale nella città di Fano, in cui la pittura murale eseguita da Orlando copre l'intero spazio absidale. L'iconografo presenta una citazione complessa di alcune figurazioni tradizionali, quali il Cristo in trono, la Gerusalemme celeste e la donna che partorisce il bambino, attingendo al consueto capitolo venticinquesimo di Matteo e ricorrendo a diversi altri richiami al libro dell'Apocalisse (in particolare ai capitoli terzo e dodicesimo).

Fra le sue numerose opere, segnalo un grande affresco dipinto per una chiesa a Lima in Perù e un San Francesco con scene della vita per il monastero delle clarisse di Oristano, in Sardegna. Attraverso di esse possiamo cogliere come questo iconografo non si limiti a ispirarsi a contenuti tradizionali ma si avvalga anche di una tavolozza tradizionale, a base di terre e colori naturali. Pur esprimendo una certa libertà le sue forme sono contenute entro un'anatomia plausibile.

Di Fabio Nones, iconografo di Trento e fondatore del Laboratorio dei Santi martiri anauniensi, presento la cosiddetta Icona ecumenica, di dimensionı monumental1 e aipınta per ı cnıesa de1 barnabiti a Bologna, e l'icona San Giuseppe con scene della vita. Il suo percorso, molto vicino a quello già menzionato di Paolo Orlando, si ispira alla tradizione per contenuti, cromie e forme grafiche.

Altro artista interessante e per certi versi vicino a questi due appena citati è l'iconografo Ivan Polverari di Roma, co-fondatore in tempi recenti dell'associazione In novitate radix. Come il nome lascia intuire, si tratta di un'istituzione che cerca di mediare fra creatività artistica e rispetto della tradizione. La familiarità di questo iconografo con la città di Roma e le icone splendide che vi sono ancora conservate è la sorgente da cui trae linfa vitale il suo percorso artistico. Presento due immagini tratte dalla sua produzione artistica: una Madre di Dio Salus populi romani e un nuovo tipo iconografico creato per rappresentare in beato Giovanni Scalabrini.

Personaggio di riferimento per quanto riguarda l'iconografia in Italia è indubbiamente Giovanni Raffa di Perugia, autore del presente libro per la parte manualistica. Porta avanti da anni una ricerca personale a partire da quella che egli chiama icona italica. Egli attribuisce questo punto di partenza alla frequentazione con 
Enzo Bianchi, che gli suggerì l'esistenza di una forma iconografica squisitamente italiana precedente il periodo delle scuole senese e fiorentina del XIII secolo e che trova in Sozio e Meliore i suoi più illustri esponenti. ${ }^{9}$ Presento un'opera da lui eseguita per una chiesa di Milano: si tratta di un antependia per altare con la scena dell'ultima cena rappresentata secondo i criteri del figurativo canonico. Raffa ha realizzato negli ultimi anni un ciclo iconografico che avvolge ormai completamente la chiesa di san Sisto a Perugia in Italia, proponendo una nuova sintesi iconografica che si richiama appunto ad una iconografia canonica rispettosa degli stilemi e dei cromatismi classici.

Accanto ai maestri iconografi che ho appena menzionato, ne indico alcuni altri (fra gli innumerevoli che si potrebbe comunque menzionare). Si tratta di fondatori di scuole iconografiche disseminate nel territorio italiano.

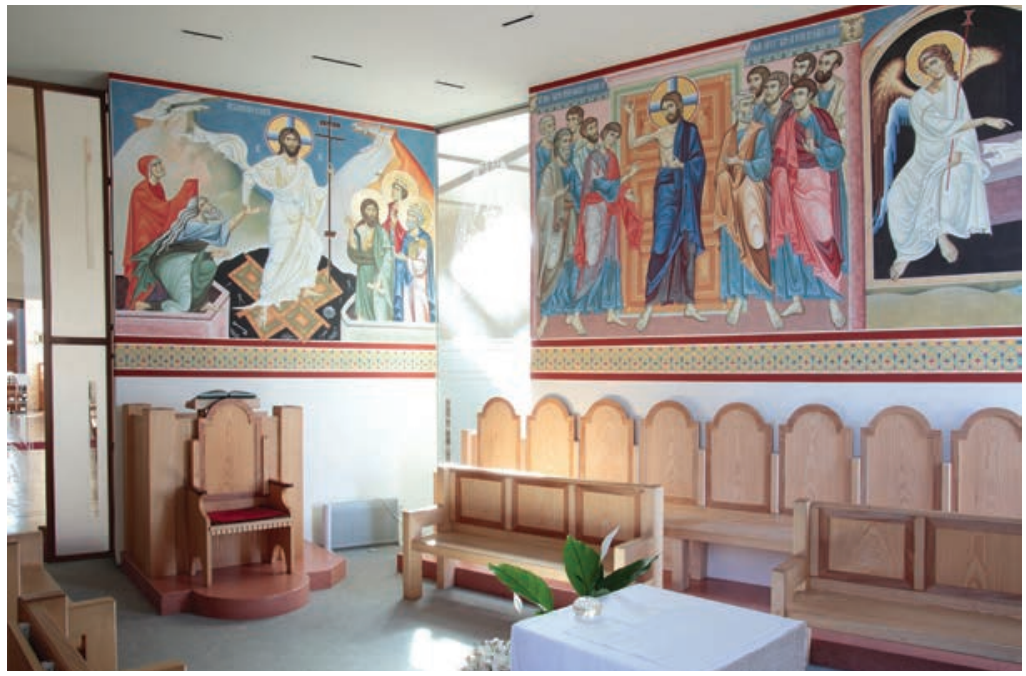

Fig.4. Chiesa di San Sisto, Perugia, Giovanni Raffa

\footnotetext{
${ }^{9}$ Enzo Bianchi, noto pubblicista e fondatore della comunità di Bose, ha dipinto diverse icone nel periodo iniziale della comunità per poi affidare all'atelier della comunità la produzione per far fronte alla domanda sempre crescente di questi manufatti.
} 


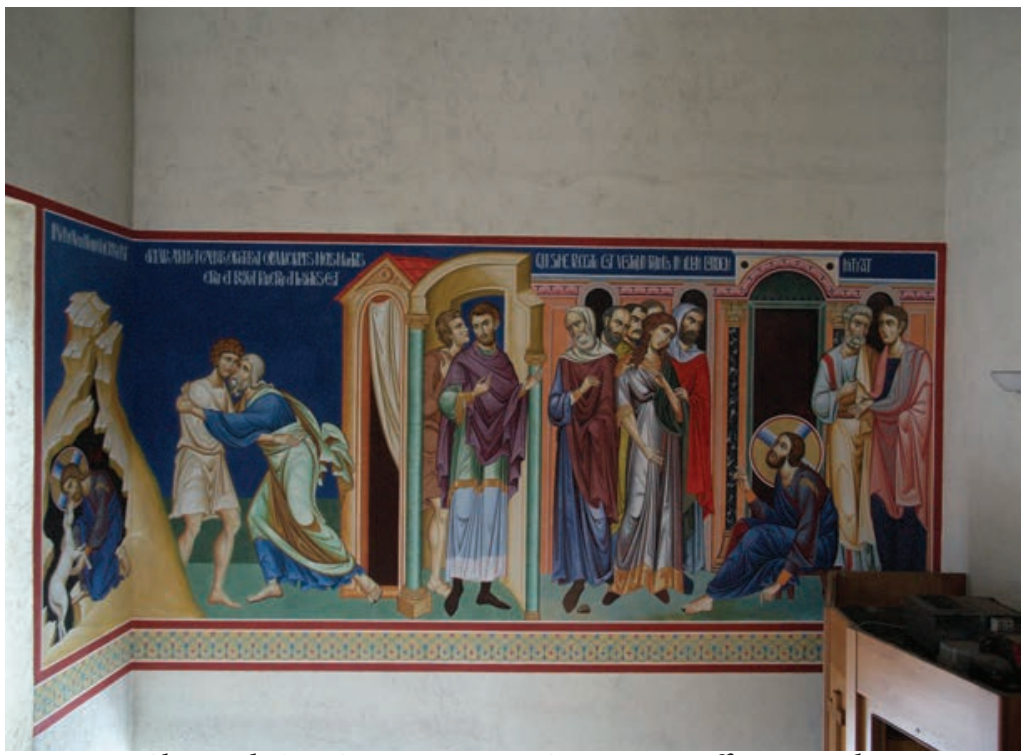

Fig.5. Chiesa di San Sisto, Perugia, Giovanni Raffa, Dettaglio

L'iconografia canonica è stata trasmessa in Italia a partire dagli anni Ottanta grazie al felice apporto di maestri russi che hanno potuto risiedere in Italia per periodi prolungati. La trasmissione deriva da incontri in cui artisti italiani sono entrati in contatto con lo spirito che accompagnava la nascente ricerca (soprattutto russa) in ambito di iconografia canonica. Fra questi maestri vorrei citare Alexandr Stal'nov di San Pietroburgo che, comunicando la tensione del suo atelier di provenienza, ha veicolato la pittura di icone nello spirito della Scuola di Mosca nel suo periodo aureo, il XV secolo.

L'attenzione per la conformità delle copie, la raffinata ricerca per un disegno esatto, la ricerca filologica per i materiali e i pigmenti naturali sono la tipica impronta lasciata da questo maestro su innumerevoli allievi italiani divenuti in breve maestri iconografi.

Di Giancarlo Pellegrini, di Bologna, presento un crocifisso dipinto ' per una chiesa monastica a Strasburgo in Francia. L'introduzione dei crocifissi collocati sopra l'altare fa pensare ad un altro 
aspetto dell'arte figurativa contemporanea per la liturgia: il recupero di quella collocazione nel tramezzo assai consueta nel Gotico. Ancora una volta la rappresentazione figurativa aderente al canone è frutto di una riscoperta della tradizione preesistente. Una riscoperta a cui spesso danno spesso voce i moderni committenti.

Vorrei citare inoltre alcuni miei lavori: la Discesa agli inferi e la Crocifissione, un'opera eseguita con l'aiuto dei detenuti per la chiesa del carcere di Bologna in stretta conformità ai modelli russi del periodo aureo del XV secolo.

Nella chiesa del Paradiso di Faenza, nella parte alta del battistero artistico eseguito dai ceramisti faentini, è stata collocata una teoria di tre grandi icone, copie conformi della scuola di Rublev.

Un'icona della Madre di Dio con $i$ santi Teresa a Massimiliano Kolbe, eseguita per la cappella delle suore missionarie a Roma. Qui la conformità è mantenuta anche se si muove nello spazio di una certa innovazione. Altro ciclo che segue i criteri appena esposti è il ciclo mariano per la cappella di san Biagio a Maranello.

\section{Alcune conclusioni}

La presentazione delle opere che aiutano a comprendere visivamente le quattro categorie (il non figurativo, la forma particolare di un non figurativo delle vetrate, il figurativo non canonico e il figurativo canonico) rilette alla luce delle cinque tesi dello Spirito della liturgia del cardinale Ratzinger conduce ad una domanda inevitabile: cosa a è concesso fare oggi nella Chiesa cattolica in materia di arte per la liturgia?

La Chiesa cattolica è aperta ad ogni possibilità artistica, perché nel corso dell'evoluzione del dogma non si è mai accolto uno stile a discapito di un altro. Sarebbe difficile pensare di bussare alle porte dell'istituzione Chiesa chiedendo che si abbandonino certe strade e se ne promuovano altre, perché si è scelta l'opzione di massima apertura alle forme espressive.

Infatti, se ci si concede di dedicarsi ad una lettura attenta del testo del cardinale Ratzinger non sfugge come ad un teologo conoscitore della tradizione, dopo l'esposizione delle cinque tesi, sembri quasi costretto a rimettersi nel solco dei documenti ufficiali e 
sia indotto ad affermare per l'assegnazione delle opere artistiche un criterio guida decisamente minimale. Nella conclusione del testo leggiamo infatti un monito che sorprende nella sua apparente genericità, là dove il cardinale si congeda dai suoi lettori affermando «chi fa opere d'arte nella Chiesa oggi, abbia fede».

Il cardinale Ratzinger giunge di fatto alla stessa conclusione pratica dei cardinali Bellarmino e Paleotti nel già citato contesto della Controriforma, quando si vide naufragare la speranza di definire le forme artistiche secondo canoni ben precisi. In quel frangente i cardinali si limitarono a esporre tre requisiti minimi per l'opera d'arte destinata ai luoghi deputati al culto. Essa infatti deve limitarsi a «non rappresentare il nudo, non creare confusione nei fedeli in materia di fede» e ispirare «alla giusta devozione». ${ }^{10}$

Il grande interrogativo, che abbiamo espresso nella domanda Che cosa è opportuno fare o non fare oggi nella Chiesa cattolica in materia di iconografia?, trova dunque risposta in una valutazione estremamente ampia e aperta ad ogni possibilità. È tuttavia molto interessante ricordare come un eminente teologo divenuto cardinale avesse già messo a fuoco interrogativi tanto pertinenti e puntuali.

Ritengo che fra le quattro categorie considerate le più interessanti siano le ultime due. Il non figurativo, tanto nella forma più astratta quanto in quella espressa attraverso le vetrate istoriate, è attualmente la strada più percorsa e la più orientata ad ottemperare un'arte funzionale al religioso o al sacro intesi in senso ampio.

Tuttavia limitandosi al senso stretto del testo è abbastanza evidente quanto queste categorie non rispettino il significato profondo né la definizione accordata al concetto peculiare di un'arte orientata alla liturgia. Ciò che lasciare perplessi, quando si considerino opere d'arte afferenti alle prime due categorie, sono soprattutto le tesi in cui si invocava il principio della figurazione come risposta al mistero dell'incarnazione.

Un'arte per la liturgia plausibile sarebbe piuttosto quella offerta dalle ultime due categorie, che optano per la necessità di un figurativo da esprimersi in forme più o meno canoniche. Un'opzione

${ }^{10}$ Daniele Menozzi, La Chiesa e le immagini. I testi fondamentali sulle arti figurative dalle origini ai giorni nostri (Torino: San Paolo, 1995), 205-228. 
per la quale si registra una marcata crescita della richiesta rispetto ai decenni scorsi.

Ancora, entrando nel merito delle ultime due categorie, vorrei segnalare una crescita della sensibilità nel modo di sentire all'occidentale che va di pari passo con la sua peculiare tradizione artistica. A causa della mancanza di canoni stretti, affermatasi soprattutto dal manierismo in poi, si è infatti creata una disponibilità implicita che ricerca figurazioni in cui la creatività si impone spesso sul canone che crea una sorta di simpatia a favore della terza categoria. Al contrario, e per gli stessi motivi, si crea sempre e comunque una certa diffidenza nei confronti della quarta categoria, che sembrerebbe limitare l'espressione artistica soggettiva a favore del rispetto del canone.

Come membro di una commissione per l'arte sacra di una grande diocesi che esamina diversi progetti ogni anno, quando vengono presentate iconografie che si ispirano a moduli canonici mi imbatto in atteggiamenti di perplessità che a volte toccano la vera e propria avversione. Casi in cui affiora il pensiero del cardinale nella quinta tesi, dove suggerisce:

La nostra teologia deve ricentrarsi sui dettami del Niceno II, non dimenticare la nostra tradizione peculiare che inizia con il gotico cercando tuttavia di non dare norme canoniche quali definizioni, come ad esempio fu per la Chiesa russa nel Concilio dei cento capitoli. ${ }^{11}$

Se teniamo in filigrana proprio la quinta tesi capiamo perché ci sia oggi molta simpatia per l'iconografia dinamica identificabile nella terza categoria (figurativo non canonico) e perché vi sia al contrario una certa forma di resistenza per quella della quarta categoria (figurativo canonico). Per un cattolico un'immagine proveniente dalla tradizione ortodossa (benché affondi spesso le radici nel periodo della chiesa indivisa) suona come derivata da un'altra tradizione, e quindi non immediatamente assimilabile con le categorie dell'arte occidentale.

${ }^{11}$ Joseph Ratzinger, Introduzione allo spirito della liturgia (Torino: San Paolo Edizioni 2014), 131. 
Queste riflessione è molto importante per l'iconografia e gli iconografi di oggi, perché spesso non si riesce a fare luce sui motivi di una certa avversione che la chiesa istituzione sembra mostrare nei confronti dell'iconografia canonica. L'intento che mi proponevo in questo capitolo era precisamente quello di aiutare ad uscire da una certa forma di miopia che non lascia comprendere l'ampio respiro delle problematiche in gioco.

Troppo spesso ciò avviene perché non si presta poca cura nel ricercare la causa dei fenomeni e ci si accontenta di sterili critiche legate a slogan o luoghi comuni. Ho voluto appunto proporre questa ricerca e confronto con lo spirito della Liturgia, non tanto per dare risposte, ma per aiutare, come di consueto, a formulare le giuste questioni, quando la ricerca (e si può dire della nostra problematica) non sia ancora matura ed incapace di dare orizzonti propositivi. Citando Aristotele, Il sapiente è colui che conosce le cause. ${ }^{12}$

Credo che il cammino sia soltanto agli inizi, la storia dell'immagine in occidente è veramente articolata e presenta tappe salienti ben identificabili tuttavia la riflessione combinata fra teologia, magistero e vita della chiesa è soltanto agli albori, perché non esistono testi scritti da autori occidentali che si siano addentrati realmente nella problematica con l'obiettivo di sviscerarla. ${ }^{13}$

Il compito dell'integrazione (se e come) della tradizione occidentale con l'ingresso dell'iconografia canonica che ci è stata mediata negli ultimi decenni dalla tradizione orientale è arduo ma possibile. Incrementare la ricerca nel tentativo di inquadrare i termini della questione in una possibile visione di sintesi è la strada che ci si apre davanti.

\section{Literature:}

Aristotele. Metafisica. Roma: Bompiani, 2000.

Busi, Gianluca. Il segno di Giona. Bolgna: Dehoniana libri, 2012.

Barral I Altet Xavier. Vetrate medievali in Europa. Milano: Jacabook, 2003.

\footnotetext{
${ }^{12}$ Aristotele, Metafisica (Roma: Bompiani, 2000).

${ }^{13}$ Gianluca Busi, Il segno di Giona, (Bolgna: Dehoniana libri, 2012), 6.
} 
Bergamo Maurizio del Prete Mattia. Spazi celebrativi. L'architettura dell'Ecclesia. Bologna: EDB, 2003.

Menozzi, Daniele. La Chiesa e le immagini. I testi fondamentali sulle arti figurative dalle origini ai giorni nostri. Torino: San Paolo, 1995.

Ratzinger, Joseph. Opera Omnia, 11: Teologia della Liturgia. Introduzione allo spirito della liturgia, 11 voll. Città del Vaticano: Ed. Vaticana, 2010.

Ratzinger, Joseph. Introduzione allo spirito della Liturgia. Torino: San Paolo Edizioni, 2014.

Rupnik, Marko I. Il colore della luce. Roma: Lipa, 2003. 


\section{ДИСАТИ ПУНИМ ПЛУЋИМА ПРАВОСЛАВНЕ ИКОНЕ И МОГУЋЕ ИНТЕРПРЕТАЦИЈЕ У КАТОЛИЧКИМ ЦРКВАМА}

Gianluca Busi

Универзизет у Болоњи, Теолошки факултет ел.пошта: sleodgianluca@alice.it

Резиме: Рад се базира на тезама изнетим у књизи Папе Бенедикта XIV „Дух литургије” а у којој се у пет основних тачака разматра могућност да се у католичкој Црква поново успостави сакрална ликовна уметност са посебним освртом на изгубљена блага црквене уметности источне цркве. С обзиром на католичке цркве изграђене после 60-их, он их дели у четри главне категорије: цркве које не прихватају ни једну фигуративну слику, затим оне које се украшавају искључиво уметничким шарама витража, оне које садрже фигуративне слике, али без поштовања традиционалног сликарског канона и оне цркве које прихватају украшавање католичких цркава фигуративим сликарством и то искљичиво канонским сликама/иконама. Коначно се долази до ове тачке гледишта: да би чак и по католичкој традицији после средњег века, а посебно након што је маниризам постаје отворен за било коју врсту уметности (па и апстрактну), данас било занимљиво промовисати нову црквену уметност кроз канонске/иконе слике. Уколико би ce ce то десило, уметнички пројекти би били респектабилни са идејом да хришћанство дише са два плућна крила источним и западном црквом, а да се црквена уметност на потпуно нови начин преобликује узимајући обе ликовне традиције као узоре, како са запада тако и са истока. Сликање икона и иконографских пројеката на овај начин би за католичку црквену уметност могао бити убедљив начин да постави своје темеље у 3. миленијуму.

Кључне речи: иконописац, иконе, литургија, црква, традиција 